\title{
QCD spectroscopy and quark mass renormalisation in external magnetic fields with Wilson fermions*
}

\author{
Gunnar Bali, Bastian B. Brandt ${ }^{\dagger}$, Gergely Endrődi and Benjamin Gläßle \\ Institute for Theoretical Physics \\ University of Regensburg \\ D-93040 Regensburg \\ E-mail: bastian.brandteur.de
}

\begin{abstract}
We study the change of the QCD spectrum of low-lying mesons in the presence of an external magnetic field using Wilson fermions in the quenched approximation. Motivated by qualitative differences observed in the spectra of overlap and Wilson fermions for large magnetic fields, we investigate the dependence of the additive quark mass renormalisation on the magnetic field. We provide evidence that the magnetic field changes the critical quark mass both in the free case and on our quenched ensemble. The associated change of the bare quark mass with the magnetic field affects the spectrum and is relevant for the magnetic field dependence of a number of related quantities. We derive Ward identities for lattice and continuum QCD+QED from which we can extract the current quark masses. We also report on a first test of the tuning of the quark masses with the magnetic field using the current quark masses, and show that this tuning resolves the qualitative discrepancy between the Wilson and overlap spectra.
\end{abstract}

The 33rd International Symposium on Lattice Field Theory

14-18 July 2015

Kobe International Conference Center, Kobe, Japan

\footnotetext{
*Acknowledgments: The simulations have been done on 'iDataCool' at the university of Regensburg. This work was supported by SFB/TRR-55. We would like to thank Kalman Szabo and Falk Bruckmann for useful discussions.

†peaker.
} 


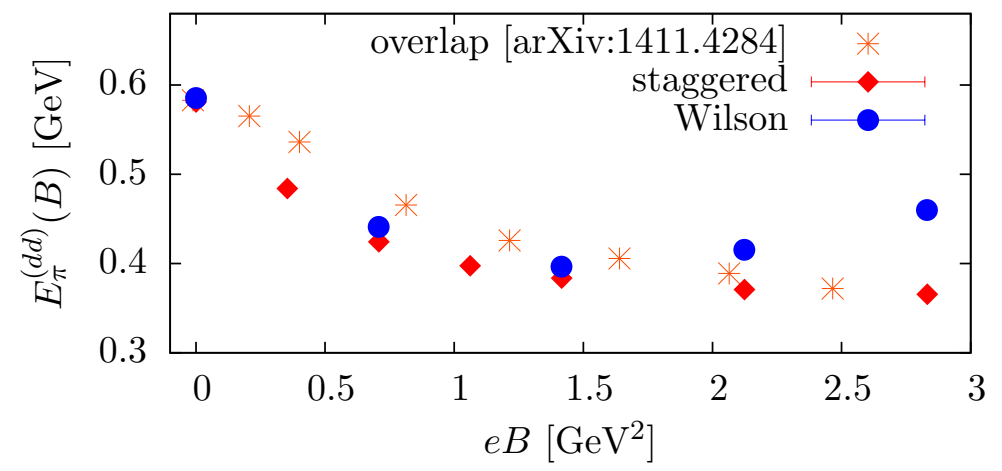

Figure 1: Comparison between results for the energies of connected neutral pions with $\bar{d} d$ flavour content with respect to the external magnetic field obtained from different fermion discretisations. The results for Wilson and staggered fermions are from the current study and the results for overlap fermions are the taken from [9]. Here the pion mass at $\mathbf{B}=\mathbf{0}$ is about $580 \mathrm{MeV}$.

\section{Introduction}

Besides the strong interest in studying the impact of QED on QCD observables (for a review see e.g. [1]), there is also a growing interest in the properties of QCD in strong external magnetic fields. They may appear in non-central heavy-ion collisions [2], inside magnetars [3] and in the evolution of the early universe [4]. It is important to note that external magnetic fields change the thermodynamic properties of $\mathrm{QCD}$, as well as the spectrum and other zero temperature characteristics. In particular, the change in the energy levels of low-lying hadrons has an impact on all the physical situations mentioned above. Furthermore, it was suggested [5] that vector mesons might become massless and condense at some critical magnetic field, leading to a superconductivity of the QCD vacuum along the magnetic field axis [6]. While the thermodynamic properties of QCD in the presence of external magnetic fields are by now rather well understood (see [7]) there are only a few initial quenched studies of the spectrum $[8,9,10,11]$. These studies suggest that $\rho$-meson condensation does not occur, although indications for the contrary have also been reported [12].

In our study we aim at investigating the spectrum of low-lying hadrons exposed to external magnetic fields $\mathbf{B}$ using Wilson fermions, initially in the quenched setup and neglecting $O(a)$ improvement. Figure 1 shows a comparison of the results for the energies of the connected neutral pions with $\bar{d} d$ flavour content, $\pi_{d d}$, obtained from Wilson and staggered fermions on our quenched ensemble and overlap fermions from [9] at a $\mathbf{B}=\mathbf{0}$ pion mass of about $580 \mathrm{MeV}$ and constant input quark mass $a \bar{m}$ (or constant hopping parameter $\kappa=1 /(2 a \bar{m}+8)$ ). In contrast to the results from other fermion discretisations, the results for Wilson fermions show a non-monotonous behaviour with increasing B. A crucial difference between Wilson fermions and the other discretisations is the presence of an additive quark mass renormalisation $a \bar{m}_{c}$. Its value depends on the properties of the Wilson term in the Dirac operator where the magnetic field enters directly via the link variables, see eq. (2.2). Consequentially, it is to be expected that $a \bar{m}_{c}$ will change with $B$, in analogy to its change in the presence of QED [1]. In fact, this change of $a \bar{m}_{c}$, and consequently the change in the bare quark masses $a_{f} \sim a\left(\bar{m}_{f}-\bar{m}_{c, f}\right)$, is already present in the free case, which we will show in section 3 . Though formally a lattice artefact, this effect may be numerically large and, affecting the neutral pion energies additively, its relative impact is enhanced towards small pion masses. This 
explains why the effect is comparably small in figure 1 , while it is much more prominent below in figure 3 (right) where the quark mass is smaller. Moreover, as this effect results in quark masses to change with the magnetic field at fixed $a \bar{m}$, quark mass and magnetic field dependence become coupled in a non-trivial way. The impact of this subtlety of Wilson fermion formulations on results obtained at finite lattice spacing has so far been ignored, see e.g. [8, 10, 11].

\section{QCD in an external magnetic field}

We consider an external magnetic field $\mathbf{B}=B \mathbf{e}_{3}$ pointing in $x_{3}$-direction, which can be generated by a vector potential of the form

$$
A_{1}(x)=-\eta B x_{2}, \quad A_{2}(x)=(1-\eta) B x_{1} \quad \text { and } \quad A_{0}(x)=A_{3}(x)=0 .
$$

Here $\eta \in[0,1]$ is a free parameter which does not enter physical observables. The following results are obtained using the symmetric gauge, $\eta=1 / 2$, but we have explicitly checked that the results agree when we set $\eta=1$. Note, that we also need to include "twists" at the boundary to satisfy periodic boundary conditions for the gauge potential and that the flux of the magnetic field is quantised according to $e B a^{2} N_{1} N_{2}=6 \pi N_{B}\left(0 \leq N_{B}<N_{1} N_{2}\right)$, where $N_{i}$ is the number of lattice points in $x_{i}$-direction and $e>0$ is the elementary charge (see, e.g., Ref. [13]). The external magnetic field enters the system via the covariant derivative by minimal coupling of $A_{\mu}$ to the quark charges $q_{f}$ as $D_{\mu} \rightarrow D_{\mu}+i q_{f} A_{\mu}$. For two flavours $\left(q_{u}=2 e / 3\right.$ and $\left.q_{d}=-e / 3\right)$, this results in the replacement of the standard lattice link variables $U_{\mu}^{\mathrm{G}}(x) \in \mathrm{SU}(3)$ in the Dirac operator by the new links (diagonal in flavour space)

$$
U_{\mu}(x)=U_{\mu}^{\mathrm{G}}(x) u_{\mu}(x) \in \mathrm{U}(3) \times \mathrm{SU}_{f}(2) \quad \text { with } \quad u_{\mu}(x)=\exp \left[i e a\left(\frac{1}{6}+\frac{\tau^{3}}{2}\right) A_{\mu}(x)\right] .
$$

In the following we employ the unimproved Wilson Dirac operator $D_{W}$ with hopping parameters $\kappa_{f}$ corresponding to input quark masses $a \bar{m}_{f}=\left(\kappa_{f}^{-1}-8\right) / 2$.

\section{The free case}

We begin the discussion with the free case, where quarks couple to the external magnetic field through their electric charges but are blind to QCD interactions. To find how the additive mass renormalisation for Wilson fermions depends on $B$, we will make use of the particular form of the relativistic energy levels (Landau-levels) for fermions $(|\mathbf{s}|=1 / 2)$ and for bosons,

$$
\text { fermions: } E_{f}^{2}(B)=m_{f}^{2}+(2 n+1)\left|q_{f} B\right|-2 \mathbf{s} \cdot q_{f} \mathbf{B}, \quad \text { bosons: } E_{f}^{2}(B)=m_{f}^{2}+(2 n+1)\left|q_{f} B\right| .
$$

The massless Wilson Dirac operator may be written schematically as $a D_{W}=a \not D+a^{2} \Delta / 2$, where $\not D$ is the anti-Hermitian part and $\Delta$ the Wilson term. While the former describes spin- $1 / 2$ particles, the latter is the discretisation of the Klein-Gordon operator and, thus, describes bosons. This difference between spin-statistics becomes relevant when we switch on the external magnetic field in both operators. Setting $m_{f}=0$ in eq. (3.1), we read off that the lowest eigenvalue of $\not D$ 

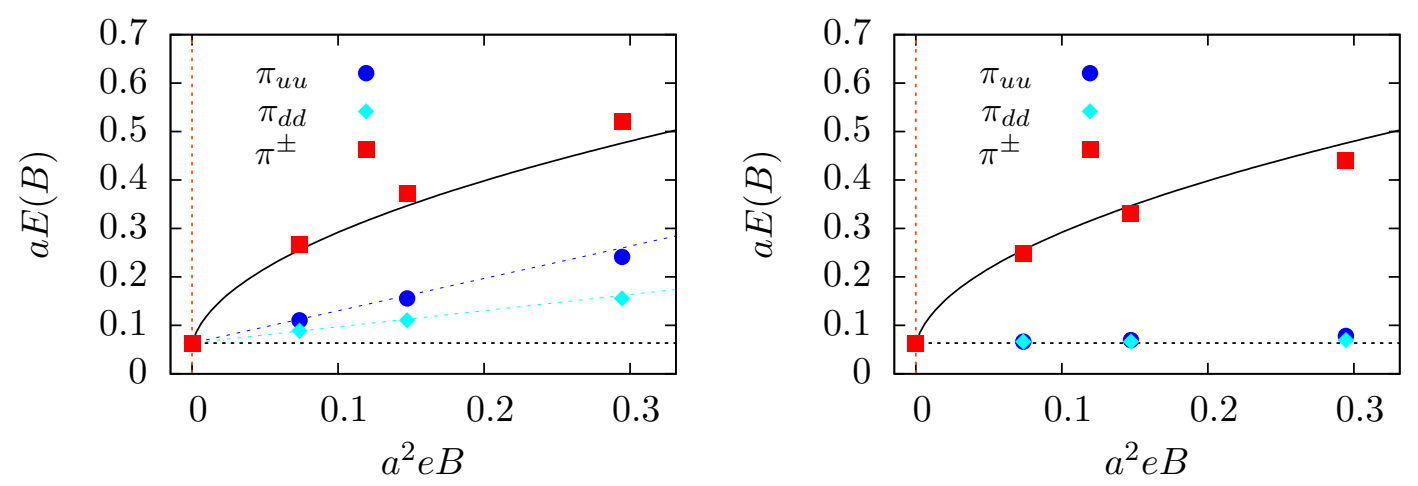

Figure 2: Results for the energies associated with free correlation functions in the pseudoscalar channel without (left) and with (right) tuning $\kappa$ with $B$ along the trajectory (3.2). The coloured dashed lines in the left plot show the analytic expectations $E_{f}(B)=E(0)+c_{f}(B)$ for energies associated with neutral correlation functions and the solid line is the expectation for charged "pions".

vanishes ( $n=0$ and $\mathbf{s} \cdot q_{f} \mathbf{B}>0$ ), while that of the Wilson term equals $a^{2}\left|q_{f} B\right| / 2$. Accordingly ${ }^{1}$, the real part of the lowest eigenvalue of $D_{W}$ does not remain zero, but increases linearly with $B$. (We checked this numerically by calculating the eigenvalues of $D_{W}$.) This increase is equivalent to an additive shift in the quark mass, implying a $B$-dependent additive renormalization. Thus, simulating at fixed quark mass $m_{f}$ is achieved by tuning the hopping parameters along the trajectory,

$$
\kappa_{f}^{-1}(B)=\kappa^{-1}(B=0)-c_{f}(B), \quad c_{f}(B) \equiv a^{2}\left|q_{f} B\right| .
$$

Note that this additive shift in the spectrum is only present for Wilson fermions and does not appear, for example, in the staggered formulation [14].

To demonstrate the effect of this $B$-dependent tuning, it is instructive to measure the free "pion masses", i.e. the energies associated to the leading decay of the pseudoscalar correlation functions, corresponding to the energies of quark-antiquark states with imposed pion quantum numbers. On the one hand, for neutral correlation functions, both quarks have $\mathbf{s} \cdot q_{f} \mathbf{B}>0$ in the ground state, so that the associated energy is $E(B)=m_{f}+m_{f^{\prime}}$, cf. eq. (3.1). On the other hand, for the charged correlation function one of the quarks (that with the smaller absolute charge) is forced to have $\mathbf{s} \cdot q_{f} \mathbf{B}<0$, and the energy is given by $E(B)=m_{f}+\sqrt{m_{f^{\prime}}^{2}+2\left|q_{f^{\prime}} B\right|}$.

Our numerical results for the energies at fixed $\kappa=0.124$ are shown in figure 2 (left). The energy of the neutral pion increases with the magnetic field, indicating the unphysical increase of the quark mass by the amount $c_{f}(B)$. Tuning the hopping parameters along the trajectory (3.2) instead, the neutral pion mass remains constant as it should, see figure 2 (right). Our results are also in good agreement with the expectation for the charged "pion".

\section{Full QCD}

\subsection{Additive quark mass renormalisation and Ward identities}

When the quarks feel the QCD interactions, the situation becomes more complicated, since

\footnotetext{
${ }^{1}$ Even though $[\not D, \Delta] \neq 0$ and thus the two operators do not share a common eigensystem, the lowest eigenmodes do coincide, as can be checked analytically in the continuum. Thus, the lowest eigenvalue of $a D_{W}$ is simply the sum of the lowest eigenvalues of $a \not \supset$ and of $a^{2} \Delta / 2$.
} 
the neutral pion mass will change with the magnetic field. However, it is known from chiral perturbation theory that even for non-zero magnetic field the energy of the neutral pion remains zero at vanishing quark mass [15], so that a chiral extrapolation of these energies allows to determine $\kappa_{c}$. In practice, the situation is even more involved since the mass eigenstates are mixings between the flavour eigenstates and $\kappa_{c}$ becomes flavour dependent, $\kappa_{c} \rightarrow \kappa_{c, f}(B)$. In addition, the neutral correlation functions obtain a contribution from disconnected diagrams, which we neglect.

An alternative is to use the current quark masses appearing in Ward-Takahashi identities [16]. In the presence of external magnetic fields the identities change, since the covariant derivatives are not proportional to the unit matrix in flavour space. In the continuum the vector and axial-vector Ward identities read (omitting the $U_{A}(1)$ anomaly term)

$$
\begin{aligned}
\partial_{\mu}\left(J_{V}\right)_{\mu}^{j}(x)= & i \varepsilon_{3 j k}\left\{\left(m_{u}-m_{d}\right) \bar{\psi}(x) \frac{\tau^{k}}{2} \psi(x)+i \bar{\psi}(x) \gamma_{\mu} A_{\mu}(x) \frac{\tau^{k}}{2} \psi(x)\right\} \\
\partial_{\mu}\left(J_{A}\right)_{\mu}^{j}(x)= & \left(m_{u}+m_{d}\right) \bar{\psi}(x) \gamma_{5} \frac{\tau^{j}}{2} \psi(x)+\delta_{j 3} \frac{1}{2}\left(m_{u}-m_{d}\right) \bar{\psi}(x) \gamma_{5} \mathbf{1} \psi(x) \\
& -\varepsilon_{3 j k} \bar{\psi}(x) A_{\mu}(x) \gamma_{\mu} \gamma_{5} \frac{\tau^{k}}{2} \psi(x) .
\end{aligned}
$$

Here $J_{V / A}$ are vector and axial-vector currents, respectively, and $\varepsilon_{i j k}$ is the totally antisymmetric tensor. ${ }^{2}$ On the lattice with Wilson fermions the Ward identities obtain similar terms and additional dimension 5 operators due to the variation of the Wilson term. A publication containing the lattice identities is in preparation [18].

For neutral correlation functions the new terms vanish, leaving the standard identities in place. Naively, one can define singlett quark masses associated with the connected neutral pions with $\bar{u} u$ and $\bar{d} d$ flavour content. However, the "neutral" correlation functions in these identities receive disconnected contributions. Furthermore, the axial identity for $j=3$ contains a mixture of $\bar{u} u$ and $\bar{d} d$ correlation functions and their separation demands the use of the identity for the unit matrix, which is violated at the quantum level due to the axial anomaly. A clean alternative is to use the identities for "charged" correlation functions. In this case no disconnected diagrams contribute. The problem, however, is that vector and axial-vector identities are needed to determine the point where $u$ and $d$ quark masses both vanish. We plan to exploit this strategy in the future.

\subsection{A test of tuning with connected neutral correlation functions}

Our quenched setup for testing uses a $48 \times 16^{3}$ lattice at $\beta=6.00$. Following [19], this results in a lattice spacing of $a \approx 0.09 \mathrm{fm}$ and the critical hopping parameter at $\mathbf{B}=\mathbf{0}$ is $\kappa_{c}=$ $1 /\left(2 a \bar{m}_{c}+8\right)=0.157131$. We have generated 200 uncorrelated configurations and performed one measurement per configuration with a Wuppertal smeared [20] source including spatially APE smeared [21] links, employing CHROMA [22].

For a first determination of the shift in the additive quark mass renormalisation in the interacting (quenched) case, we extract $\kappa_{c, f}(B)$ using the current quark masses $m_{f}^{\mathrm{WI}}$ associated with the Ward-Takahashi identities including neutral correlation functions. We determine $m_{f}^{\mathrm{WI}}$ from

$$
m_{f}^{\mathrm{WI}}=\frac{\partial_{0}\left\langle\left(J_{A}^{f f}\right)_{0}\left(x_{0}\right) P^{f f}(0)\right\rangle}{\left\langle P^{f f}\left(x_{0}\right) P^{f f}(0)\right\rangle}
$$

\footnotetext{
${ }^{2}$ For the axial Ward identity in the continuum and for domain wall fermions on the lattice see [17].
} 

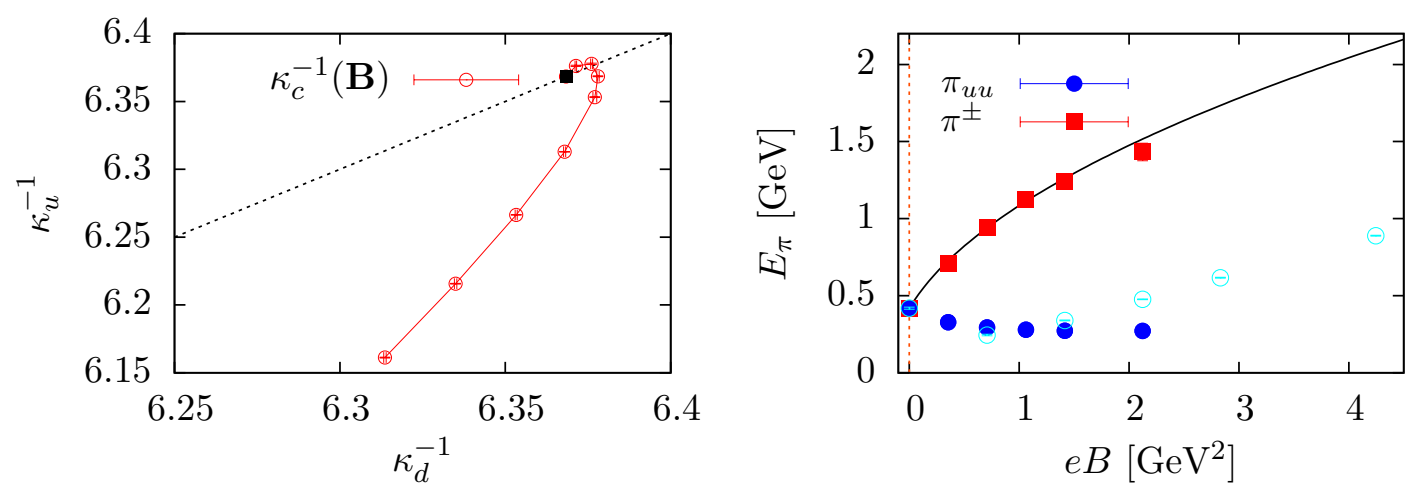

Figure 3: Left: Results for the critical hopping parameters $\kappa_{c, f}$, for $f=u, d$, for different values of $B$, as a trajectory in the plane of inverse hopping parameters. The startpoint at $B=0$ is the black point on the symmetric axis (dashed line). Right: Results for the pion spectrum versus the strength of the external magnetic field with $\kappa_{u}$ and $\kappa_{d}$ tuned to achieve a constant bare quark mass. The black line shows the lowest Landau level prediction for the charged pions and the open circles display the $\pi_{u u}$ masses obtained from constant $\kappa$ for comparison.

for several values of $\kappa$ (we have results for neutral pion energies down to $300 \mathrm{MeV}$ for all values of $B$ ) and perform a linear chiral extrapolation to the point where $m_{f}^{\mathrm{WI}}=0$ for each value of $B$. In eq. (4.3) $\partial_{\mu}$ is the backward lattice derivative, $J_{A}$ is the point-split axial vector current and the superscript $f f$ indicates neutral operators. The details will be contained in our future publication. Note, that we obtain fully compatible results when we extrapolate the connected neutral pion energies instead.

The results for $\kappa_{c, f}$ are shown as a trajectory in the plane of inverse $u$ and $d$ quark hopping parameters in figure 3 (left). Their values reflect the non-monotonous behaviour of the neutral pion energies seen in figure 3 (right), leading to a curl in the trajectory. Using these results we now retune the hopping parameter with $B$, to achieve a constant bare quark mass, and look at the effect on the spectrum. The results for the pion energies obtained with constant bare quark masses, corresponding to a pion mass of about $400 \mathrm{MeV}$ at $\mathbf{B}=\mathbf{0}$, are shown in figure 3 (right). For comparison we have also shown the energies obtained when $\kappa=\kappa_{u}=\kappa_{d}$ is kept constant. Comparing the two sets of results, we see that the retuning of the bare quark masses disposes of the non-monotonous behaviour of the neutral pion masses and, consequentially, the discrepancy with the results from other fermion discretisations.

\section{Conclusions and perspectives}

In this proceedings article we have reported on our ongoing study on the spectrum of low lying hadrons exposed to strong external magnetic fields with Wilson fermions. Figure 1 shows a discrepancy in the large $B$ behaviour of connected neutral pions between results from Wilson fermions and other fermion discretisations. We have shown that (at least parts of) this discrepancy is due to a change in the additive renormalisation of the quark mass $\bar{m}_{c, f}$ and the associated change in the bare quark mass with $B$. We have shown that this effect is present already in the free case and that it stems from the bosonic nature of the Wilson-term in the action. It can be removed by tuning the hopping parameter as a function of $B$. The associated change of the bare and renormalised 
quark mass affects all quantities that are sensitive to changes in $m_{f}$ by (potentially large) lattice artefacts. Accordingly, some caution is required when comparing quantities evaluated at different magnetic fields at finite lattice spacing.

Since the pion energies change with the magnetic field, they cannot be used for a direct tuning of quark masses. We thus propose to use the current quark mass associated to lattice WardTakahashi identities in QCD+QED (the Wilson fermion identities will be published in [18]) for the tuning and reported on a first test using neutral correlation functions. Apart from changes in the mutliplicative renormalisation, the associated current quark masses provide a means to compare the renormalised quark masses at $B \neq 0$ with the ones at $B=0$. They can potentially also be used for a clean definition and extraction of quark masses in QCD+QED.

\section{References}

[1] A. Portelli, PoS LATTICE 2014 (2015) 013 [arXiv:1505.07057].

[2] D. Kharzeev, Phys. Lett. B 633 (2006) 260 [hep-ph/0406125]; D. E. Kharzeev, L. D. McLerran and H. J. Warringa, Nucl. Phys. A 803 (2008) 227 [arXiv:0711.0950].

[3] E. J. Ferrer and V. de la Incera, Lect. Notes Phys. 871 (2013) 399 [arXiv:1208.5179].

[4] T. Vachaspati, Phys. Lett. B 265 (1991) 258.

[5] S. Schramm, B. Müller and A. J. Schramm, Mod. Phys. Lett. A 7 (1992) 973.

[6] M. N. Chernodub, Phys. Rev. Lett. 106 (2011) 142003 [arXiv:1101.0117].

[7] K. Szabó, PoS LATTICE 2013 (2014) 014 [arXiv:1401.4192]; M. D’Elia, PoS LATTICE 2014 (2015) 020 [arXiv:1502.06047].

[8] Y. Hidaka and A. Yamamoto, Phys. Rev. D 87 (2013) 9, 094502 [arXiv:1209.0007].

[9] E. V. Luschevskaya et al, Nucl. Phys. B 898 (2015) 627 [arXiv:1411.4284].

[10] S. R. Beane et al., Phys. Rev. Lett. 113 (2014) 25, 252001 [arXiv:1409.3556].

[11] W. Detmold et al, arXiv:1508.05884.

[12] V. V. Braguta et al, Phys. Lett. B 718 (2012) 667 [arXiv:1104.3767].

[13] G. S. Bali et al, JHEP 1202, 044 (2012) [arXiv:1111.4956 [hep-lat]].

[14] G. Endrődi, PoS LATTICE 2014 (2014) 018 [arXiv:1410.8028].

[15] N. O. Agasian and I. A. Shushpanov, JHEP 0110 (2001) 006 [hep-ph/0107128].

[16] M. Bochicchio et al, Nucl. Phys. B 262 (1985) 331.

[17] T. Blum et al, Phys. Rev. D 76 (2007) 114508 [arXiv:0708.0484].

[18] G. Bali, B.B. Brandt, G. Endrődi and B. Gläßle, in preparation.

[19] T. Bhattacharya et al, Phys. Rev. D 53 (1996) 6486 [hep-lat/9512021].

[20] S. Güsken, Nucl. Phys. Proc. Suppl. 17 (1990) 361.

[21] M. Falcioni et al, Nucl. Phys. B 251 (1985) 624.

[22] R. G. Edwards et al., Nucl. Phys. Proc. Suppl. 140 (2005) 832 [hep-lat/0409003]. 